\title{
Emerging epidemic dog rabies in coastal South Africa: A molecular epidemiological analysis
}

\author{
Peter Coetzee $^{\mathrm{a}}$ and Louis H. Nel ${ }^{\mathrm{a}}$ \\ ${ }^{\mathrm{a}}$ Department of Microbiology and Plant Pathology, University of Pretoria, 0001 \\ Pretoria, South Africa
}

\begin{abstract}
Towards understanding the molecular epidemiology of a severe dog rabies epidemic in the KwaZulu Natal province of South Africa, we analyzed a variable 592 nucleotide genome sequence domain of 170 rabies viruses from KwaZulu Natal and surrounding regions. Viruses from the KwaZulu Natal and Eastern Cape provinces belonged to a unique lineage, circulating as two independent and expanding epidemiological cycles. The first presented as closely related dog cycles along the eastern coastal regions of the two provinces, while the second, in northern KwaZulu Natal, has entered into at least one wildlife reservoir, the black backed jackal. We underline the success and opportunism of rabies in southern Africa, in a likely reflection of the emergence and radiation of rabies in new host species and locales throughout the larger continent as a whole.
\end{abstract}

\section{Article Outline}

1. Introduction

2. Materials and methods

2.1. Viruses

2.2. cDNA synthesis, PCR amplification and sequencing

2.3. Phylogenetic analysis

3. Results

4. Discussion 
Acknowledgements

References

\section{Introduction}

Rabies virus (RABV) is one of the most widespread animal viruses and continues to scourge virtually the entire globe as the agent of severe and lethal encephalopathy of all terrestrial mammals, including man (Rupprecht et al., 2002, Swanepoel, 2005 and WHO, 2004). RABV belongs to genotype 1 of the Lyssavirus genus of the family Rhabdoviridae, order Mononegavirales and contains a single negative sense RNA genome of approximately $12 \mathrm{~kb}$. In southern Africa, two biotypes of RABV are maintained independently among the members of the Herpestidae and Canidae families, respectively (Von Teichman et al., 1995 and Nel et al., 2005). Canid rabies is relatively new to the southern African subcontinent, having been introduced from infectious cycles that had existed among domestic dogs in Angola in the 1940s (Swanepoel, 2005). It has since spread widely throughout the Republic of South Africa (RSA), with the increasing involvement of wildlife, including bat eared foxes (Otocyon megalotis) in the western regions of the country, and black backed jackal (Canis mesomelas) in the northern Limpopo province (Bishop et al., 2003, Sabeta et al., 2003 and Swanepoel, 2005). However, in recent decades, the majority of animal and human rabies cases in RSA consistently occurred in the province of KwaZulu Natal (KZN), where the disease has become endemic in the large population of domestic dogs (Canis familiaris) of this province (Bishop et al., 2003 and Swanepoel, 2005). There is a close correlation between the number of cases in dogs, with those in humans and livestock, and the control of dog rabies remains the single most important factor in minimizing the public and veterinary health consequences of rabies here, as it is throughout Africa and in most of the rest of the developing world.

KZN is located on the eastern seaboard of RSA, and is one of the smallest $\left(92,100 \mathrm{~km}^{2}\right)$ and the most populated (approximately 9.5 million people) of the nine RSA provinces. It extends from the international borders with Swaziland and Mozambique in the north, to 
the province of the Eastern Cape (EC) in the south, while inland it is bound by the provinces of the Freestate and Mpumalanga, and by the Kingdom of Lesotho (Fig. 1). Two dog rabies epidemics, believed to have originated from dog endemic regions which had existed in southern Mozambique since 1952, have occurred among domestic dogs in KZN (Bishop et al., 2003 and Swanepoel, 2005). The first of these epidemics started in 1964, and apparently without spreading into the adjacent EC, ended by 1968, due to mass vaccination and strict dog control (Bishop et al., 2003 and Swanepoel, 2005). The second epidemic, which started in 1976, coincided with the outbreak of civil war in Mozambique and the fleeing of refugees across the international border with RSA (KZN). This epidemic has proven to be intractable despite the concerted control efforts of the South African Directorate of Veterinary Services (Bishop et al., 2003 and Swanepoel, 2005) and is thought to have penetrated far and wide into the province and adjacent regionsrabies has for example been reported from the EC since 1987 (Bishop et al., 2003 and Swanepoel, 2005). Reasons for the persistence of the second epidemic are varied, but of significant importance is an increase in urbanization since the late 1980's, mostly due to social and political changes. The development of numerous informal settlements around towns and cities followed and large dog populations capable of maintaining the disease were rapidly introduced (Kloeck, 1993). Subsequent logistical, financial and managerial difficulties in the control of dog movement and in the implementation and maintenance of successful vaccination strategies, contribute to the persistence of the epidemic (Randles, 2003 and Perret, 2005). 


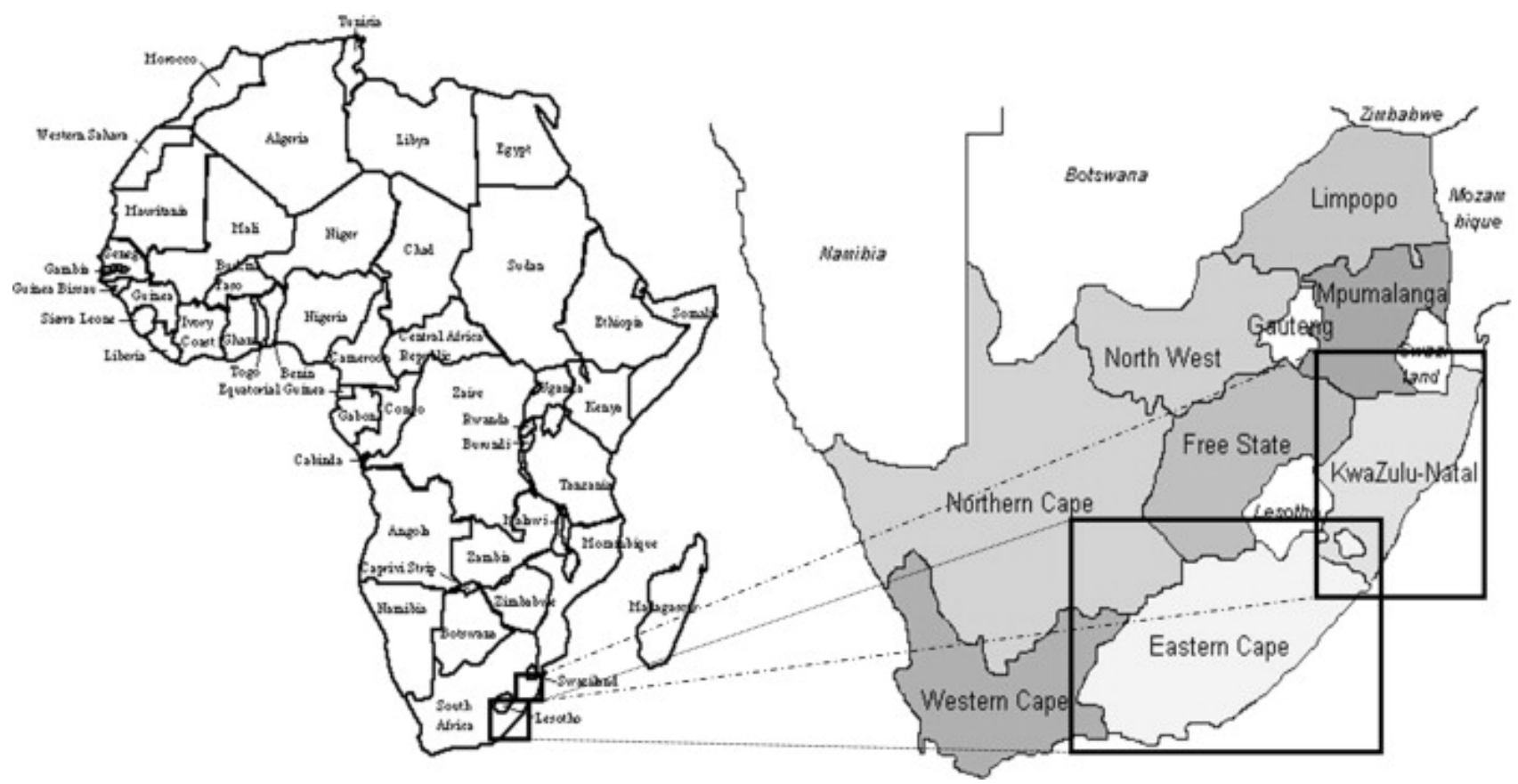

Fig. 1. Map of Africa and South Africa indicating the geographic location of the KwaZulu Natal and Eastern Cape provinces.

A better understanding of the molecular epidemiology of this growing rabies epidemic should assist in future surveillance and control efforts and towards this objective, we conducted a molecular sequence analysis of a representative panel of viruses, obtained from the KZN province during a given calendar year (2003). Previous molecular epidemiological studies in southern Africa included a small number of isolates from $\mathrm{KZN}$, but were primarily aimed at illustrating the distinction between the canid and mongoose biotypes of the disease (King, 1993, Von Teichman et al., 1995 and Nel et al., 1997), or were focused on determining the genetic diversity of the canid biotype over a broader geographical region (Sabeta et al., 2003). Here our emphasis was placed on determining the regional genetic variation of RABV within $K Z N$, and on delineating the course of the epidemic through the different magisterial districts of the province.

Subsequently, we assessed the relationship of this epidemic with cycles from elsewhere in southern Africa. Our analysis was based on the carboxyl terminal domain of the glycoprotein (G, cytoplasmic domain) and the G-L intergenic region (L representing the downstream 'large' viral polymerase gene), which constitute the most variable portion of 
the RABV genome (Tordo and Kouknetzoff, 1993). This target was considered appropriate for distinguishing closely related viral variants from each other, as it has been shown in various studies to be well suited for the investigation of the molecular epidemiology of rabies in defined geographical domains (Tordo et al., 1986, Sacramento et al., 1991, Nel et al., 1993, Nadin-Davis, 2000 and Paez et al., 2003).

In this study we present the first molecular epidemiological evidence for the emerging distribution patterns of rabies in coastal southern Africa and resolve an epidemiological scenario that may well be typical for much of the African continent.

\section{Materials and methods}

\subsection{Viruses}

Details of viruses analyzed are presented in Table 1. During the year 2003, 235 animals from KZN were diagnosed as rabies positive with a standard immunofluorescent assay (Bishop et al., 2003). Samples of infected brain material were stored at $-20{ }^{\circ} \mathrm{C}$ in $50 \%$ glycerol-phosphate buffered saline (PBS) without further passage (Allerton Veterinary Laboratories, Pietermaritzburg, RSA). Of these, 123 samples that represented each of the affected magisterial districts of KZN, were selected for further analysis. Also included were 19 samples from animal cases in EC (Onderstepoort Veterinary Research Institute, Pretoria, RSA). 
Table 1.

Panel of rabies viruses from the KwaZulu Natal (KZN) and the Eastern Cape (EC) provinces of South Africa

\begin{tabular}{|c|c|c|c|c|c|c|c|}
\hline $\begin{array}{l}\text { Virus } \\
\text { number }\end{array}$ & $\begin{array}{l}\text { Allerton reference } \\
\text { number }^{\mathrm{a}}\end{array}$ & Species $^{b}$ & Country/province & $\begin{array}{l}\text { Magisterial } \\
\text { district }\end{array}$ & $\begin{array}{l}\text { Year of } \\
\text { isolation }\end{array}$ & $\begin{array}{l}\text { Grid } \\
\text { reference } \\
\text { number }^{c}\end{array}$ & $\begin{array}{l}\text { Genbank } \\
\text { accession } \\
\text { number }\end{array}$ \\
\hline 1 & KZNcw03.83 & Bovine & South Africa/KwaZulu Natal & Alfred & 2003 & G17 & DQ841424 \\
\hline 2 & KZNdg03.89 & Canine & South Africa/KwaZulu Natal & Pongola & 2003 & L4 & DQ841427 \\
\hline 3 & KZNcw03.103 & Bovine & South Africa/KwaZulu Natal & Pongola & 2003 & L4 & DQ841425 \\
\hline 4 & KZNdg03.105 & Canine & South Africa/KwaZulu Natal & Eshowe & 2003 & L10 & DQ841428 \\
\hline 5 & KZNdg03.106 & Canine & South Africa/KwaZulu Natal & Lower Umfolozi & 2003 & O9 & DQ841429 \\
\hline 6 & KZNdg03.114 & Canine & South Africa/KwaZulu Natal & Mount Currie & 2003 & D17 & DQ841430 \\
\hline 7 & KZNdg03.120 & Canine & South Africa/KwaZulu Natal & Alfred & 2003 & H17 & DQ841431 \\
\hline 8 & KZNdg03.121 & Canine & South Africa/KwaZulu Natal & Alfred & 2003 & G17 & DQ841432 \\
\hline 9 & KZNdg03.127 & Canine & South Africa/KwaZulu Natal & Ixopo & 2003 & G15 & DQ841433 \\
\hline 10 & KZNdg03.129 & Canine & South Africa/KwaZulu Natal & Inanda & 2003 & K13 & DQ841434 \\
\hline 11 & KZNdg03.133 & Canine & South Africa/KwaZulu Natal & Lower Tugela & 2003 & L12 & DQ841435 \\
\hline 12 & KZNdg03.137 & Canine & South Africa/KwaZulu Natal & Hlabisa & 2003 & P7 & DQ841436 \\
\hline 13 & KZNdg03.142 & Canine & South Africa/KwaZulu Natal & Durban & 2003 & K14 & DQ841437 \\
\hline
\end{tabular}




\begin{tabular}{|c|c|c|c|c|c|c|c|}
\hline $\begin{array}{l}\text { Virus } \\
\text { number }\end{array}$ & $\begin{array}{l}\text { Allerton reference } \\
\text { number }^{\mathrm{a}}\end{array}$ & Species $^{b}$ & Country/province & $\begin{array}{l}\text { Magisterial } \\
\text { district }\end{array}$ & $\begin{array}{l}\text { Year of } \\
\text { isolation }\end{array}$ & $\begin{array}{l}\text { Grid } \\
\text { reference } \\
\text { number }^{c}\end{array}$ & $\begin{array}{l}\text { Genbank } \\
\text { accession } \\
\text { number }\end{array}$ \\
\hline 14 & KZNdg03.147 & Canine & South Africa/KwaZulu Natal & Umzinto & 2003 & $\mathrm{I} 17$ & DQ841438 \\
\hline 15 & KZNdg03.149 & Canine & South Africa/KwaZulu Natal & Umvoti & 2003 & I1 1 & DQ841439 \\
\hline 16 & KZNdg03.152 & Canine & South Africa/KwaZulu Natal & Ndwedwe & 2003 & $\mathrm{~J} 13$ & DQ841440 \\
\hline 17 & KZNdg03.169 & Canine & South Africa/KwaZulu Natal & Eshowe & 2003 & L10 & DQ841441 \\
\hline 18 & KZNdg03.170 & Canine & South Africa/KwaZulu Natal & Vryheid & 2003 & K6 & DQ841442 \\
\hline 19 & KZNdg03.176 & Canine & South Africa/KwaZulu Natal & Vryheid & 2003 & K6 & DQ841443 \\
\hline 20 & KZNdg03.180 & Canine & South Africa/KwaZulu Natal & Lower Umfolozi & 2003 & P6 & DQ841444 \\
\hline 21 & KZNdg03.192 & Canine & South Africa/KwaZulu Natal & Port Shepstone & 2003 & H18 & DQ841445 \\
\hline 22 & KZNdg03.194 & Canine & South Africa/KwaZulu Natal & Lower Tugela & 2003 & K12 & DQ841446 \\
\hline 23 & KZNdg03.200 & Canine & South Africa/KwaZulu Natal & Lower Tugela & 2003 & L12 & DQ841447 \\
\hline 24 & KZNDg03.204 & Canine & South Africa/KwaZulu Natal & Lower Tugela & 2003 & L11 & DQ841448 \\
\hline 25 & KZNdg03.205 & Canine & South Africa/KwaZulu Natal & Pietemaritzburg & 2003 & H13 & DQ841449 \\
\hline 26 & KZNdg03.209 & Canine & South Africa/KwaZulu Natal & Lower Umfolozi & 2003 & N9 & DQ841450 \\
\hline 27 & KZNdg03.213 & Canine & South Africa/KwaZulu Natal & Port Shepstone & 2003 & H17 & DQ841451 \\
\hline
\end{tabular}




\begin{tabular}{|c|c|c|c|c|c|c|c|}
\hline $\begin{array}{l}\text { Virus } \\
\text { number }\end{array}$ & $\begin{array}{l}\text { Allerton reference } \\
\text { number }^{\mathrm{a}}\end{array}$ & Species $^{b}$ & Country/province & $\begin{array}{l}\text { Magisterial } \\
\text { district }\end{array}$ & $\begin{array}{l}\text { Year of } \\
\text { isolation }\end{array}$ & $\begin{array}{l}\text { Grid } \\
\text { reference } \\
\text { number }^{c}\end{array}$ & $\begin{array}{l}\text { Genbank } \\
\text { accession } \\
\text { number }\end{array}$ \\
\hline 28 & KZNdg03.214 & Canine & South Africa/KwaZulu Natal & Pongola & 2003 & L4 & DQ841452 \\
\hline 29 & KZNdg03.215 & Canine & South Africa/KwaZulu Natal & Hlabisa & 2003 & $\mathrm{O} 7$ & DQ841453 \\
\hline 30 & KZNdg03.225 & Canine & South Africa/KwaZulu Natal & Umzimkulu & 2003 & E16 & DQ841454 \\
\hline 31 & KZNdg03.230 & Canine & South Africa/KwaZulu Natal & Vryheid & 2003 & $\mathrm{~J} 6$ & DQ841455 \\
\hline 32 & KZNdg03.235 & Canine & South Africa/KwaZulu Natal & Inanda & 2003 & $\mathrm{~J} 13$ & DQ841456 \\
\hline 33 & KZNdg03.236 & Canine & South Africa/KwaZulu Natal & Alfred & 2003 & F17 & DQ841457 \\
\hline 34 & KZNdg03.237 & Canine & South Africa/KwaZulu Natal & Ndwedwe & 2003 & $\mathrm{~J} 13$ & DQ841458 \\
\hline 35 & KZNdg03.241 & Canine & South Africa/KwaZulu Natal & Mhlabatini & 2003 & L8 & DQ841459 \\
\hline 36 & KZNdg03.247 & Canine & South Africa/KwaZulu Natal & Vryheid & 2003 & $\mathrm{~J} 6$ & DQ841460 \\
\hline 37 & KZNdg03.251 & Canine & South Africa/KwaZulu Natal & Ndwedwe & 2003 & $\mathrm{~J} 13$ & DQ841461 \\
\hline 38 & KZNdg03.253 & Canine & South Africa/KwaZulu Natal & Vyheid & 2003 & $\mathrm{~J} 6$ & DQ841462 \\
\hline 39 & KZNdg03.254 & Canine & South Africa/KwaZulu Natal & Lower Umfolozi & 2003 & N9 & DQ841463 \\
\hline 40 & KZNdg03.256 & Canine & South Africa/KwaZulu Natal & Pinetown & 2003 & $\mathrm{~J} 14$ & DQ841464 \\
\hline 41 & KZNdg03.263 & Canine & South Africa/KwaZulu Natal & Inanda & 2003 & $\mathrm{~J} 13$ & DQ841465 \\
\hline
\end{tabular}




\begin{tabular}{|c|c|c|c|c|c|c|c|}
\hline $\begin{array}{l}\text { Virus } \\
\text { number }\end{array}$ & $\begin{array}{l}\text { Allerton reference } \\
\text { number }^{\mathrm{a}}\end{array}$ & Species $^{b}$ & Country/province & $\begin{array}{l}\text { Magisterial } \\
\text { district }\end{array}$ & $\begin{array}{l}\text { Year of } \\
\text { isolation }\end{array}$ & $\begin{array}{l}\text { Grid } \\
\text { reference } \\
\text { number }^{c}\end{array}$ & $\begin{array}{l}\text { Genbank } \\
\text { accession } \\
\text { number }\end{array}$ \\
\hline 42 & KZNdg03.264 & Canine & South Africa/KwaZulu Natal & Ngotshe & 2003 & L5 & DQ841466 \\
\hline 43 & KZNdg03.265 & Canine & South Africa/KwaZulu Natal & Pinetown & 2003 & $\mathrm{~J} 14$ & DQ841464 \\
\hline 44 & KZNdg03.269 & Canine & South Africa/KwaZulu Natal & Vryheid & 2003 & $\mathrm{~J} 5$ & DQ841468 \\
\hline 45 & KZNdg03.270 & Canine & South Africa/KwaZulu Natal & Eshowe & 2003 & M10 & DQ841469 \\
\hline 46 & KZNdg03.276 & Canine & South Africa/KwaZulu Natal & Nwedwe & 2003 & $\mathrm{~J} 13$ & DQ841470 \\
\hline 47 & KZNdg03.290 & Canine & South Africa/KwaZulu Natal & Vryheid & 2003 & J6 & DQ841471 \\
\hline 48 & KZNdg03.292 & Canine & South Africa/KwaZulu Natal & Hlabisa & 2003 & $\mathrm{O} 7$ & DQ841472 \\
\hline 49 & KZNdg03.293 & Canine & South Africa/KwaZulu Natal & Durban & 2003 & $\mathrm{~J} 14$ & DQ841473 \\
\hline 50 & KZNdg03.299 & Canine & South Africa/KwaZulu Natal & Durban & 2003 & K14 & DQ841474 \\
\hline 51 & KZNdg03.302 & Canine & South Africa/KwaZulu Natal & Eshowe & 2003 & L10 & DQ841475 \\
\hline 52 & KZNdg03.306 & Canine & South Africa/KwaZulu Natal & Lower Umfolozi & 2003 & N9 & DQ841476 \\
\hline 53 & KZNdg03.307 & Canine & South Africa/KwaZulu Natal & Hlabisa & 2003 & $\mathrm{O} 7$ & DQ841477 \\
\hline 54 & KZNdg03.308 & Canine & South Africa/KwaZulu Natal & Lower Umfolozi & 2003 & N9 & DQ841478 \\
\hline 55 & KZNdg03.309 & Canine & South Africa/KwaZulu Natal & Lower Umfolozi & 2003 & N9 & DQ841479 \\
\hline
\end{tabular}




\begin{tabular}{|c|c|c|c|c|c|c|c|}
\hline $\begin{array}{l}\text { Virus } \\
\text { number }\end{array}$ & $\begin{array}{l}\text { Allerton reference } \\
\text { number }^{\mathrm{a}}\end{array}$ & Species $^{b}$ & Country/province & $\begin{array}{l}\text { Magisterial } \\
\text { district }\end{array}$ & $\begin{array}{l}\text { Year of } \\
\text { isolation }\end{array}$ & $\begin{array}{l}\text { Grid } \\
\text { reference } \\
\text { number }^{c}\end{array}$ & $\begin{array}{l}\text { Genbank } \\
\text { accession } \\
\text { number }\end{array}$ \\
\hline 56 & KZNdg03.314 & Canine & South Africa/KwaZulu Natal & Hlabisa & 2003 & O8 & DQ841480 \\
\hline 57 & KZNdg03.315 & Canine & South Africa/KwaZulu Natal & Vryheid & 2003 & $\mathrm{~J} 6$ & DQ841481 \\
\hline 58 & KZNdg03.316 & Canine & South Africa/KwaZulu Natal & Nkandhla & 2003 & J9 & DQ841482 \\
\hline 59 & KZNdg03.321 & Canine & South Africa/KwaZulu Natal & Ngotshe & 2003 & L5 & DQ841483 \\
\hline 60 & KZNdg03.326 & Canine & South Africa/KwaZulu Natal & Lower Umfolozi & 2003 & N9 & DQ841484 \\
\hline 61 & KZNdg03.328 & Canine & South Africa/KwaZulu Natal & Lower Umfolozi & 2003 & M4 & DQ841485 \\
\hline 62 & KZNgt03.330 & Caprine & South Africa/KwaZulu Natal & Lower Umfolozi & 2003 & N9 & DQ841542 \\
\hline 63 & KZNdg03.335 & Canine & South Africa/KwaZulu Natal & Vryheid & 2003 & J5 & DQ841486 \\
\hline 64 & KZNdg03.336 & Canine & South Africa/KwaZulu Natal & $?$ & 2003 & $?$ & DQ841487 \\
\hline 65 & KZNdg03.340 & Canine & South Africa/KwaZulu Natal & Lower Umfolozi & 2003 & N9 & DQ841488 \\
\hline 66 & KZNdg03.343 & Canine & South Africa/KwaZulu Natal & Mhlabatini & 2003 & L7 & DQ841489 \\
\hline 67 & KZNgt03.358 & Caprine & South Africa/KwaZulu Natal & Mhlabatini & 2003 & M7 & DQ841543 \\
\hline 68 & KZNdg03.359 & Canine & South Africa/KwaZulu Natal & Hlabisa & 2003 & $\mathrm{O} 7$ & DQ841490 \\
\hline 69 & KZNdg03.360 & Canine & South Africa/KwaZulu Natal & Pongola & 2003 & M4 & DQ841491 \\
\hline
\end{tabular}




\begin{tabular}{|c|c|c|c|c|c|c|c|}
\hline $\begin{array}{l}\text { Virus } \\
\text { number }\end{array}$ & $\begin{array}{l}\text { Allerton reference } \\
\text { number }^{\mathrm{a}}\end{array}$ & Species $^{b}$ & Country/province & $\begin{array}{l}\text { Magisterial } \\
\text { district }\end{array}$ & $\begin{array}{l}\text { Year of } \\
\text { isolation }\end{array}$ & $\begin{array}{l}\text { Grid } \\
\text { reference } \\
\text { number }^{c}\end{array}$ & $\begin{array}{l}\text { Genbank } \\
\text { accession } \\
\text { number }\end{array}$ \\
\hline 70 & KZNgt03.364 & Caprine & South Africa/KwaZulu Natal & Pongola & 2003 & L4 & DQ841544 \\
\hline 71 & KZNdg03.366 & Canine & South Africa/KwaZulu Natal & Port Shepstone & 2003 & H18 & DQ841492 \\
\hline 72 & KZNdg03.368 & Canine & South Africa/KwaZulu Natal & Lower Umfolozi & 2003 & O9 & DQ841493 \\
\hline 73 & KZNdg03.375 & Canine & South Africa/KwaZulu Natal & Eshowe & 2003 & J6 & DQ841494 \\
\hline 74 & KZNdg03.378 & Canine & South Africa/KwaZulu Natal & Port Shepstone & 2003 & H17 & DQ841495 \\
\hline 75 & KZNdg03.382 & Canine & South Africa/KwaZulu Natal & Ixopo & 2003 & G15 & DQ841496 \\
\hline 76 & KZNdg03.387 & Canine & South Africa/KwaZulu Natal & Durban & 2003 & $\mathrm{~J} 14$ & DQ841497 \\
\hline 77 & KZNdg03.389 & Canine & South Africa/KwaZulu Natal & Vryheid & 2003 & J6 & DQ841498 \\
\hline 78 & KZNdg03.391 & Canine & South Africa/KwaZulu Natal & Umzinto & 2003 & $\mathrm{I} 16$ & DQ841499 \\
\hline 79 & KZNdg03.399 & Canine & South Africa/KwaZulu Natal & Umzinto & 2003 & $\mathrm{I} 16$ & DQ841500 \\
\hline 80 & KZNdg03.400 & Canine & South Africa/KwaZulu Natal & Hlabisa & 2003 & $\mathrm{O} 7$ & DQ841501 \\
\hline 81 & KZNdg03.404 & Canine & South Africa/KwaZulu Natal & Ixopo & 2003 & G15 & DQ841502 \\
\hline 82 & KZNdg03.406 & Canine & South Africa/KwaZulu Natal & Inanda & 2003 & K13 & DQ841503 \\
\hline 83 & KZNdg03.407 & Canine & South Africa/KwaZulu Natal & Ubombo & 2003 & O5 & DQ841504 \\
\hline
\end{tabular}




\begin{tabular}{|c|c|c|c|c|c|c|c|}
\hline $\begin{array}{l}\text { Virus } \\
\text { number }\end{array}$ & $\begin{array}{l}\text { Allerton reference } \\
\text { number }^{\mathrm{a}}\end{array}$ & Species $^{b}$ & Country/province & $\begin{array}{l}\text { Magisterial } \\
\text { district }\end{array}$ & $\begin{array}{l}\text { Year of } \\
\text { isolation }\end{array}$ & $\begin{array}{l}\text { Grid } \\
\text { reference } \\
\text { number }^{c}\end{array}$ & $\begin{array}{l}\text { Genbank } \\
\text { accession } \\
\text { number }\end{array}$ \\
\hline 84 & KZNdg03.409 & Canine & South Africa/KwaZulu Natal & Mount Currie & 2003 & E17 & DQ841505 \\
\hline 85 & KZNdg03.410 & Canine & South Africa/KwaZulu Natal & Eshowe & 2003 & L10 & DQ841506 \\
\hline 86 & KZNdg03.411 & Canine & South Africa/KwaZulu Natal & Vryheid & 2003 & J6 & DQ841507 \\
\hline 87 & KZNdg03.417 & Canine & South Africa/KwaZulu Natal & Hlabisa & 2003 & $\mathrm{O} 7$ & DQ841508 \\
\hline 88 & KZNdg03.418 & Canine & South Africa/KwaZulu Natal & Port Shepstone & 2003 & H17 & DQ841509 \\
\hline 89 & KZNdg03.425 & Canine & South Africa/KwaZulu Natal & Vryheid & 2003 & L6 & DQ841510 \\
\hline 90 & KZNdg03.430 & Canine & South Africa/KwaZulu Natal & Nongoma & 2003 & N6 & DQ841511 \\
\hline 91 & KZNdg03.431 & Canine & South Africa/KwaZulu Natal & Hlabisa & 2003 & $\mathrm{O} 7$ & DQ841512 \\
\hline 92 & KZNshp03.433 & Ovine & South Africa/KwaZulu Natal & Vryheid & 2003 & J6 & DQ841403 \\
\hline 93 & KZNdg03.437 & Canine & South Africa/KwaZulu Natal & Lower Umfolozi & 2003 & O9 & DQ841513 \\
\hline 94 & KZNdg03.453 & Canine & South Africa/KwaZulu Natal & Durban & 2003 & $\mathrm{~J} 14$ & DQ841514 \\
\hline 95 & KZNdg03.454 & Canine & South Africa/KwaZulu Natal & Nongoma & 2003 & M5 & DQ841515 \\
\hline 96 & KZNdg03.455 & Canine & South Africa/KwaZulu Natal & Vryheid & 2003 & K7 & DQ841516 \\
\hline 97 & KZNdg03.461 & Canine & South Africa/KwaZulu Natal & Lower Tugela & 2003 & K12 & DQ841517 \\
\hline
\end{tabular}




\begin{tabular}{|c|c|c|c|c|c|c|c|}
\hline $\begin{array}{l}\text { Virus } \\
\text { number }\end{array}$ & $\begin{array}{l}\text { Allerton reference } \\
\text { number }^{\mathrm{a}}\end{array}$ & Species $^{b}$ & Country/province & $\begin{array}{l}\text { Magisterial } \\
\text { district }\end{array}$ & $\begin{array}{l}\text { Year of } \\
\text { isolation }\end{array}$ & $\begin{array}{l}\text { Grid } \\
\text { reference } \\
\text { number }^{c}\end{array}$ & $\begin{array}{l}\text { Genbank } \\
\text { accession } \\
\text { number }\end{array}$ \\
\hline 98 & KZNdg03.463 & Canine & South Africa/KwaZulu Natal & Lower Umfolozi & 2003 & N9 & DQ841518 \\
\hline 99 & KZNcw03.467 & Bovine & South Africa/KwaZulu Natal & Ubombo & 2003 & O5 & DQ841545 \\
\hline 100 & $\mathrm{KZNdg} 03.470$ & Canine & South Africa/KwaZulu Natal & Lower Umfolozi & 2003 & N9 & DQ841519 \\
\hline 101 & KZNdg03.474 & Canine & South Africa/KwaZulu Natal & Mount Currie & 2003 & D17 & DQ841520 \\
\hline 102 & KZNdg03.475 & Canine & South Africa/KwaZulu Natal & Lower Umfolozi & 2003 & N9 & DQ841521 \\
\hline 103 & KZNdg03.478 & Canine & South Africa/KwaZulu Natal & Lower Umfolozi & 2003 & N9 & DQ841522 \\
\hline 104 & KZNdg03.485 & Canine & South Africa/KwaZulu Natal & Mapumulo & 2003 & K11 & DQ841523 \\
\hline 105 & KZNdg03.491 & Canine & South Africa/KwaZulu Natal & Port Shepstone & 2003 & H17 & DQ841524 \\
\hline 106 & KZNdg03.492 & Canine & South Africa/KwaZulu Natal & Port Shepstone & 2003 & H17 & DQ841525 \\
\hline 107 & KZNdg03.494 & Canine & South Africa/KwaZulu Natal & Lower Umfolozi & 2003 & O9 & DQ841526 \\
\hline 108 & KZNdg03.502 & Canine & South Africa/KwaZulu Natal & Port Shepstone & 2003 & H16 & DQ841527 \\
\hline 109 & KZNdg03.503 & Canine & South Africa/KwaZulu Natal & Ingwavuma & 2003 & Q3 & DQ841528 \\
\hline 110 & KZNdg03.507 & Canine & South Africa/KwaZulu Natal & Lower Umfolozi & 2003 & N9 & DQ841529 \\
\hline 111 & KZNdg03.509 & Canine & South Africa/KwaZulu Natal & Lower Umfolozi & 2003 & N9 & DQ841530 \\
\hline
\end{tabular}




\begin{tabular}{|c|c|c|c|c|c|c|c|}
\hline $\begin{array}{l}\text { Virus } \\
\text { number }\end{array}$ & $\begin{array}{l}\text { Allerton reference } \\
\text { number }^{\mathrm{a}}\end{array}$ & Species $^{b}$ & Country/province & $\begin{array}{l}\text { Magisterial } \\
\text { district }\end{array}$ & $\begin{array}{l}\text { Year of } \\
\text { isolation }\end{array}$ & $\begin{array}{l}\text { Grid } \\
\text { reference } \\
\text { number }^{c}\end{array}$ & $\begin{array}{l}\text { Genbank } \\
\text { accession } \\
\text { number }\end{array}$ \\
\hline 112 & KZNdg03.510 & Canine & South Africa/KwaZulu Natal & Lower Umfolozi & 2003 & N9 & DQ841531 \\
\hline 113 & KZNdg03.513 & Canine & South Africa/KwaZulu Natal & Eshowe & 2003 & L10 & DQ841532 \\
\hline 114 & KZNdg03.514 & Canine & South Africa/KwaZulu Natal & Lower Umfolozi & 2003 & N9 & DQ841533 \\
\hline 115 & KZNdg03.518 & Canine & South Africa/KwaZulu Natal & Ubombo & 2003 & P6 & DQ841534 \\
\hline 116 & KZNdg03.568 & Canine & South Africa/KwaZulu Natal & Lower Umfolozi & 2003 & N9 & DQ841535 \\
\hline 117 & KZNdg03.583 & Canine & South Africa/KwaZulu Natal & Vryheid & 2003 & J6 & DQ841536 \\
\hline 118 & KZNdg03.588 & Canine & South Africa/KwaZulu Natal & Hlabisa & 2003 & P7 & DQ841537 \\
\hline 119 & KZNdg03.589 & Canine & South Africa/KwaZulu Natal & Vryheid & 2003 & K7 & DQ841538 \\
\hline 120 & KZNdg03.594 & Canine & South Africa/KwaZulu Natal & Lower Umfolozi & 2003 & N9 & DQ841539 \\
\hline 121 & KZNcw03.620 & Bovine & South Africa/KwaZulu Natal & Port Shepstone & 2003 & H17 & DQ841426 \\
\hline 122 & KZNdg03.621 & Canine & South Africa/KwaZulu Natal & Hlabisa & 2003 & O8 & DQ841540 \\
\hline 123 & KZNdg03.672 & Canine & South Africa/KwaZulu Natal & Lower Umfolozi & 2003 & $\mathrm{O} 10$ & DQ841541 \\
\hline 124 & ECdg03.05 & Canine & South Africa/Eastern Cape & Elliott & 2003 & $*$ & DQ841404 \\
\hline 125 & ECdg03.28 & Canine & South Africa/Eastern Cape & Lupapasi & 2003 & $*$ & DQ841406 \\
\hline
\end{tabular}




\begin{tabular}{|c|c|c|c|c|c|c|c|}
\hline $\begin{array}{l}\text { Virus } \\
\text { number }\end{array}$ & $\begin{array}{l}\text { Allerton reference } \\
\text { number }^{\mathrm{a}}\end{array}$ & Species $^{b}$ & Country/province & $\begin{array}{l}\text { Magisterial } \\
\text { district }\end{array}$ & $\begin{array}{l}\text { Year of } \\
\text { isolation }\end{array}$ & $\begin{array}{l}\text { Grid } \\
\text { reference } \\
\text { number }\end{array}$ & $\begin{array}{l}\text { Genbank } \\
\text { accession } \\
\text { number }\end{array}$ \\
\hline 126 & ECdg03.91 & Canine & South Africa/Eastern Cape & $\begin{array}{l}\text { Haga Haga, } \\
\text { Everest }\end{array}$ & 2003 & $*$ & DQ841408 \\
\hline 127 & ECdg03.179 & Canine & South Africa/Eastern Cape & Elliott & 2003 & $*$ & DQ841410 \\
\hline 128 & ECdg03.180 & Canine & South Africa/Eastern Cape & Elliott & 2003 & $*$ & DQ841411 \\
\hline 129 & $\mathrm{ECdg} 03.336$ & Canine & South Africa/Eastern Cape & Bayview & 2003 & $*$ & DQ841413 \\
\hline 130 & ECdg03.377 & Canine & South Africa/Eastern Cape & Lower Nququ & 2003 & $*$ & DQ841415 \\
\hline 131 & ECdg03.567 & Canine & South Africa/Eastern Cape & Ncanaseni & 2003 & $*$ & DQ841418 \\
\hline 132 & ECdg03.730 & Canine & South Africa/Eastern Cape & Ncambedlana & 2003 & $*$ & DQ841419 \\
\hline 133 & ECdg03.751 & Canine & South Africa/Eastern Cape & Vidgesville & 2003 & $*$ & DQ841420 \\
\hline 134 & ECdg03.779 & Canine & South Africa/Eastern Cape & All Saints & 2003 & $*$ & DQ841421 \\
\hline 135 & ECdg03.936 & Canine & South Africa/Eastern Cape & Butterworth & 2003 & $*$ & DQ841422 \\
\hline 136 & ECdg04.25 & Canine & South Africa/Eastern Cape & Ngangelizwe & 2004 & $*$ & DQ841405 \\
\hline 137 & ECdg04.43 & Canine & South Africa/Eastern Cape & Upper Ngqwaba & 2004 & $*$ & DQ841407 \\
\hline 138 & ECdg04.111 & Canine & South Africa/Eastern Cape & Centuli Location & 2004 & $*$ & DQ841409 \\
\hline 139 & ECdg04.218 & Canine & South Africa/Eastern Cape & North Crest & 2004 & $*$ & DQ841412 \\
\hline
\end{tabular}




\begin{tabular}{|l|l|l|l|l|l|l|l|}
\hline $\begin{array}{l}\text { Virus } \\
\text { number }\end{array}$ & $\begin{array}{l}\text { Allerton reference } \\
\text { number }^{\mathbf{a}}\end{array}$ & Species & Country/province & - & $\begin{array}{l}\text { Magisterial } \\
\text { district }\end{array}$ & $\begin{array}{l}\text { Year of } \\
\text { isolation }\end{array}$ & $\begin{array}{l}\text { Grid } \\
\text { reference } \\
\text { number }\end{array}$ \\
\hline & $-\begin{array}{l}\text { Genbank } \\
\text { accession } \\
\text { number }\end{array}$ \\
\hline 140 & ECdg04.376 & Canine & South Africa/Eastern Cape & Sterkspruit & 2004 & $*$ & - \\
\hline 141 & ECdg04.377 & Canine & South Africa/Eastern Cape & Ugie & 2004 & $*$ & DQ841414 \\
\hline 142 & ECdg04.499 & Canine & South Africa/Eastern Cape & Barkley East & 2004 & $*$ & DQ841416 \\
\hline
\end{tabular}

${ }^{a}$ Lab reference numbers: viruses from the Allerton Regional Veterinary Laboratory (KZN) are named using the zero-three or zerofour designation.

b We use the term 'canine' to refer to domestic dogs (C. familiaris).

${ }^{c}$ The approximate regions from where isolates were obtained, were indicated on a map of the KwaZulu Natal province by using a grid system, as implemented on the isolate submission forms of the Allerton Regional Veterinary Laboratory (KZN). Similar grid reference numbers were not available for isolates from the Eastern Cape. 
2.2. cDNA synthesis, PCR amplification and sequencing

Total RNA was extracted from infected brain material with Trizol reagent (Promega) and cDNA synthesized as described previously (Von Teichman et al., 1995 and Sabeta et al., 2003). PCR was performed (GeneAmp PCR 2700) in a $50 \mu$ l reaction consisting of $1 \mu 1$ cDNA, $5 \mu 1$ 10X PCR buffer (Promega), $100 \mu \mathrm{M}$ each of the four dNTPs (Promega), 10 pmol each of the $\mathrm{G}(+)$ and $\mathrm{L}(-)$ primers, and $1 \mathrm{U}$ Taq DNA polymerase (Promega). The primers (Integrated DNA Technologies, RSA) have been described by Sacramento et al. (1991). The PCR cycle reaction was as follows: $2 \mathrm{~min}, 94^{\circ} \mathrm{C} ; 30$ cycles of $94{ }^{\circ} \mathrm{C} / 50 \mathrm{~s}$, $45^{\circ} \mathrm{C} / 90 \mathrm{~s}, 72{ }^{\circ} \mathrm{C} / 60 \mathrm{~s} ; 7 \mathrm{~min}, 72{ }^{\circ} \mathrm{C}$. Amplicons were analyzed by agarose gel electrophoresis and purified with a Wizard ${ }^{\circledR} \mathrm{SV}$ kit (Promega). Cycle sequencing reactions were performed with the V 3.1 BigDye Terminator system (PE Applied Biosystems) together with the $\mathrm{G}(+)$ primer, after which unincorporated labeled ddNTPs were removed by ethanol precipitation. The reactions were resolved on an ABI 3100 DNA sequencer and sequences were trimmed with the Bioedit V.7.0 sequence alignment editor (Hall, 1999). All sequences were submitted to an international sequence repository (Genbank accession numbers; Table 1).

\subsection{Phylogenetic analysis}

Analyses were based on the continuous 592-nucleotide sequences, aligned with CLUSTALW (Thompson et al., 1994), as a subroutine of the Bioedit sequence alignment editor (Hall, 1999). Genetic distances were calculated with Kimura's two parameter method (MEGA3; Kumar et al., 2004), and used for the construction of two neighbourhood-joining (NJ) trees by the method of Saitou and Nei (1987). Confidence values for the tree topologies were evaluated with a bootstrap analysis of 1000 pseudoreplicate datasets, and considered meaningful for phylogenetic groupings when above 70\% (Hills and Bull, 1993). The results were validated by maximum likelihood analysis (Swofford and Olsen, 1990). 


\section{Results}

The vast majority of the 235 positive rabies cases identified in 2003 in KZN occurred among domestic dogs (85\%). These cases were not evenly distributed through the province, but were primarily localized in the more densely populated coastal regions. KZN is divided into 51 independently governed magisterial districts and it was convenient to use this divisioning system towards obtaining a representative virus sampling. We selected approximately half of the rabies virus samples from each magisterial district, arguing that this strategy would ensure sufficient representation from all of the affected areas of the province.

The G-L intergenic sequence target offered us satisfactory resolution, and it was found that the viruses from KZN shared an approximate intrinsic sequence identity of $98.9 \%$ (Kimura 2-parameter model, mean pairwise distance data not shown). All of these viruses were also closely related to other members of the RSA canid biotype, from elsewhere in southern Africa. However, on average the KZN viruses differed by $19.1 \%$ from the corresponding sequence of the PV genome, and by 29.4\% from a classical mongoose biotype RABV isolate (m669.90; Nel et al., 2005). Comparable results were obtained with the inclusion of sequence data from the EC viruses (results not shown), and all viruses from KZN and EC were concluded to conform to the canid biotype (Von Teichman et al., 1995). Our viruses also lacked the first of two hypothetical transcription termination and polyadenylation signals, found to be present for the G gene of the ERA and PV genomes. The absence of this signal has previously been demonstrated for street viruses from Europe as well as from South Africa and Zimbabwe (Wunner, 2002 and Sabeta et al., 2003).

Two phylogenetic trees were constructed in order to address different aspects of this epidemic. The first (Fig. 2), was constructed with all the RABV sequences from KZN $(n=123)$, and was aimed at demonstrating the evolutionary relationships between viruses from the different magisterial districts of the province. The epidemiological data of the viruses are provided in Table 1 and the corresponding location of the rabies cases are shown in Fig. 4. Our phylogenetic analyses demonstrated the existence of two major 
KZN RABV subfamilies, designated A and B. The division between these subfamilies was characterized by a mean nucleotide sequence divergence of $1.9 \%$, and was supported by a bootstrap value of $100 \%$. Subfamily A was by far the largest (composed of 102 viruses) and clearly represented the principal dog rabies cycle of KZN. Viruses belonging to this subfamily were primarily encountered in the eastern coastal districts of KZN, stretching from the border with southern Mozambique all along the coast to southern KZN. Subfamily A could be divided into two groups, the first of which (group I) consisted of seven geographically influenced sequence clusters (KZN/A/V1-KZN/A/V7, Fig. 2). Some of these clusters were also not monophyletic, in which case the subdivisions always corresponded to highly localized outbreaks of the disease (e.g. KZN/A/V3/cl1-cl2, Fig. 2). The second group of subfamily A (group II) consisted of a single sequence cluster, made up of only three viruses, found on the southernmost border of KZN with the EC province. Considering its phylogenetic and geographical distinction, this group was thought to represent a cycle distinct from the main KZN epidemic. 


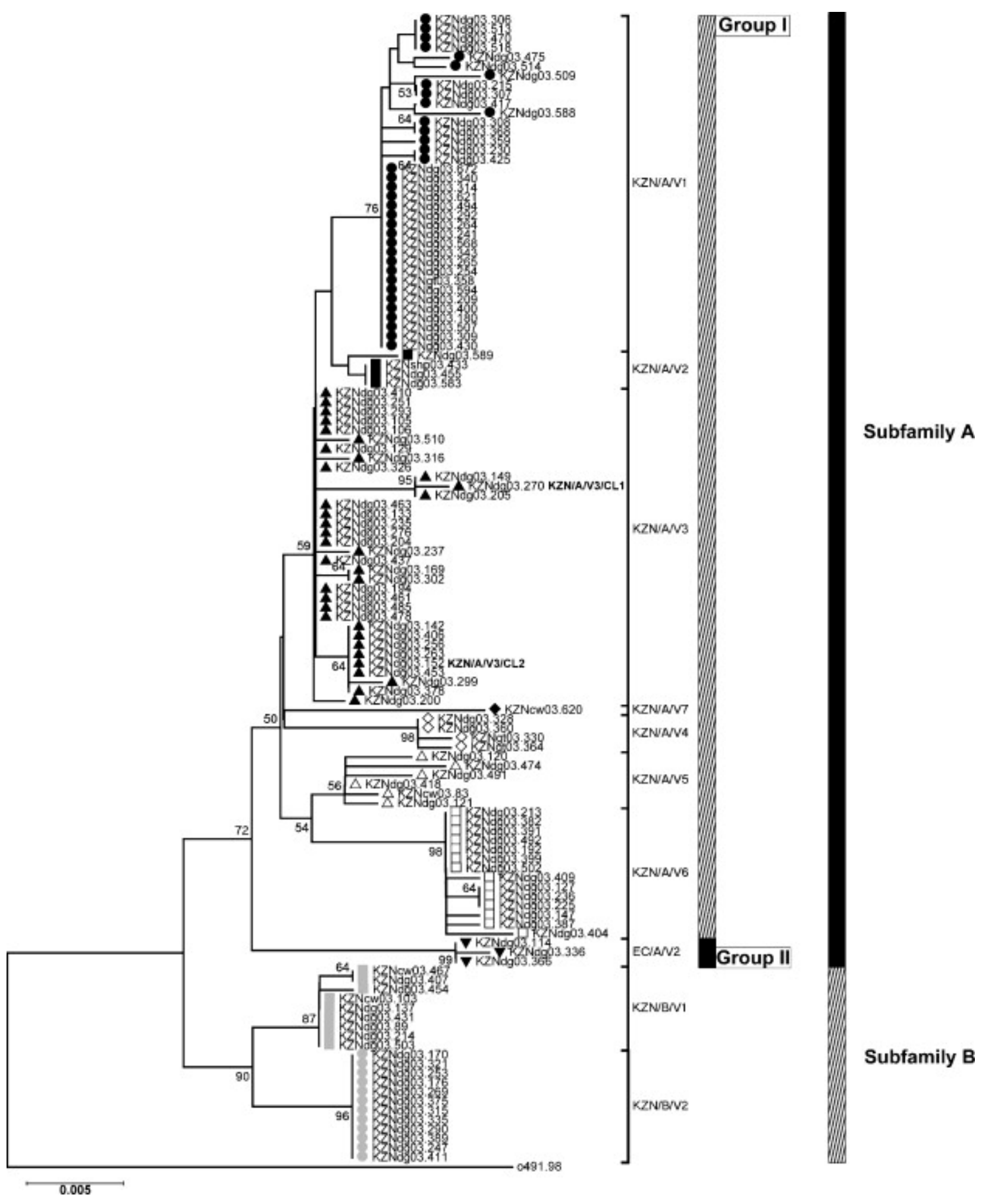

Fig. 2. Neighbourhood-joining tree of 123 nucleotide sequences of the cytoplasmic domain of the glycoprotein, and G-L intergenic region, for canine and domestic livestock 
rabies viruses from KwaZulu Natal. Horizontal branch lengths are proportional to the similarity of the sequences within and between groups, with the scale indicating the amount of nucleotide sequence divergence in substitutions per site and the vertical lines being provided for purposes of clarity only. The cognate nucleotide sequence of a bat eared fox isolate (0491.98) was used as reference sequence to root the tree. Subfamily and group divisions are indicated as discussed in the text, with virus numbers being preceded by a prefix indicating the geographic region (KZN, KwaZulu Natal) as well as host species of isolation (dg, dog/cw, bovine/gt, caprine/o, bat eared fox). 


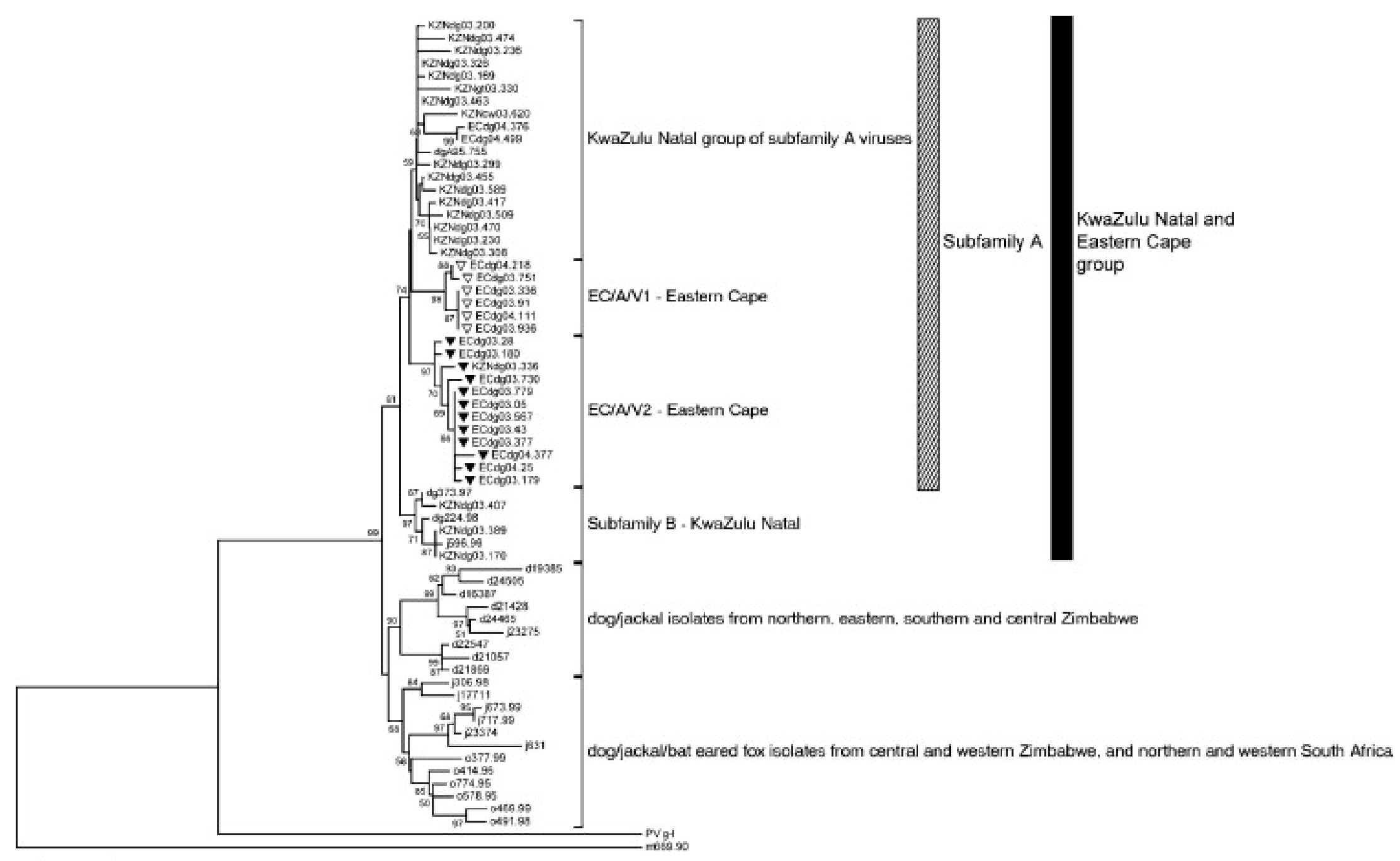

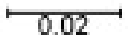

Fig. 3. Neighbourhood-joining tree of 64 nucleotide sequences of the cytoplasmic domain, and G-L intergenic region, for canine, domestic livestock and wildlife rabies viruses from KwaZulu Natal $(n=20)$, the Eastern Cape $(n=19)$, and rabies endemic regions 
from elsewhere in South Africa and Zimbabwe $(n=25)$. Horizontal branch lengths are proportional to the similarity of the sequences within and between groups, with the scale indicating the amount of nucleotide sequence divergence in substitutions per site and the vertical lines being provided for purposes of clarity only. The cognate nucleotide sequences of the PV strain and a classical mongoose rabies virus isolate (m669.90) were used as reference sequences and to root the tree (Tordo et al., 1986, Tordo et al., 1988 and Von Teichman et al., 1995). Isolate numbers are preceded by prefixes indicating the geographic region (KZN, KwaZulu Natal/EC, Eastern Cape) as well as host species of isolation ( $\mathrm{dg}(\mathrm{d}), \mathrm{dog} / \mathrm{cw}$, bovine/gt, caprine/j, jackal/o, bat eared fox). 


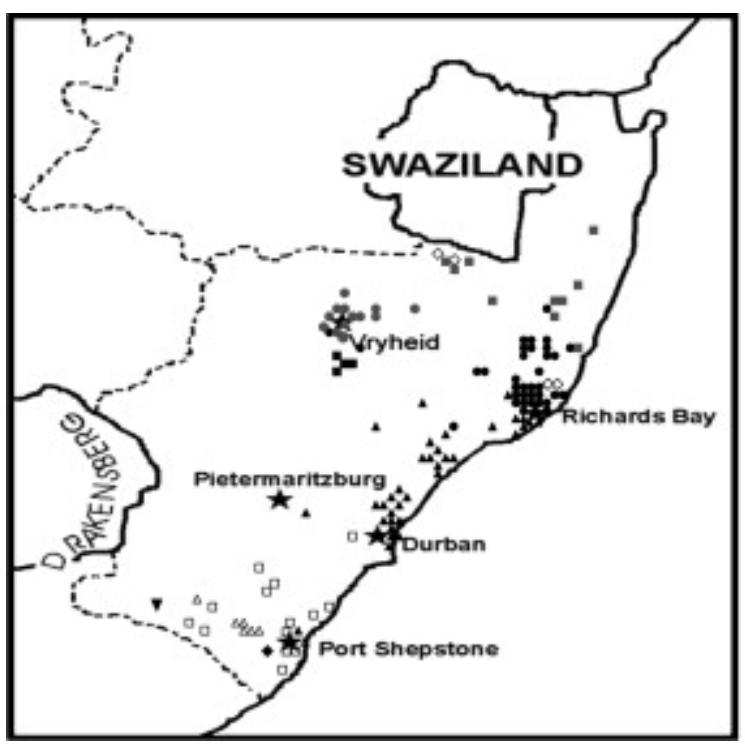

Yariant designation

Sublamiy A - Blind

KZNINN1 - •

KENINV2 - -

KEVINA : *

KEVIAVIS - 0

KONAN5 - a

KONAAVE - I

KENVWN7 - -

ECINV2 - v

Subtamly B - Gey

KENEN1 - "

KeNariz -

Fig. 4. A map of the KwaZulu Natal (KZN) province demonstrating the approximate geographic origin of rabies viruses that were sequenced during the course of this study. Symbols correspond to those that were used to indicate the respective viral groupings on the phylogenetic tree in Fig. 2.

The 21 viruses in subfamily B were very closely related (identity 99.5\%), and were all encountered in the northern regions of the province bordering the southeastern Mpumalanga province and the international border with southern Swaziland (Fig. 4). This subfamily was nevertheless composed of two distinct clusters (KZN/B/V1-KZN/B/V2), representing localized outbreaks in this geographical domain (bootstrap value 90\%, Fig. 2).

As a subsequent step in our study, we wanted to investigate the relationship of the rabies epidemic in KZN with the epidemiology of the disease in surrounding areas of South Africa. We also considered the role of wildlife canid species to be of particular importance in the larger epidemiological picture. Although rabies was first reported in 1893 from the neighbouring EC province, following an outbreak that was initiated by an infected dog imported from Britain, this outbreak was likely to have been eradicated without spillover into wildlife (Swanepoel, 2005). Since that outbreak, rabies incidenceif present in the EC, was not well documented, although the disease is known to have occurred with increasing frequency since at least 1987 . Therefore, our next phylogenetic 
analysis was based on selected viruses from KZN $(n=20)$, viruses from the adjacent province of the $\mathrm{EC}(n=19)$ (Fig. 5), and reference RABV sequences $(n=25)$ that were obtained from canine rabies endemic regions from elsewhere in RSA and Zimbabwe (Table 2). It was apparent (Fig. 3) that the viruses from the KZN and EC provinces were, as a unique cluster, distinguishable from all the other RABV isolates included in this analysis. Within this KZN and EC group, the subfamilies A and B, as described previously for the KZN viruses, were again evident. Notably, all of the viruses that were obtained from the EC province belonged to subfamily A, implying a closer relationship with these coastal region viruses than with the viruses of subfamily B. Even so, the EC viruses and the KZN viruses are separable within the subfamily A, suggesting a common introduction followed by a degree of independent evolution (Fig. 3). Significantly, RABV isolates from jackals and dogs (dg373.97, dg224.98, j596.99) that were obtained from provinces to the north of KZN (southeastern Mpumalanga; Fig. 4), clustered within the subfamily B collection of viruses, previously shown as viruses specific to northern KZN (bootstrap, 97\%).

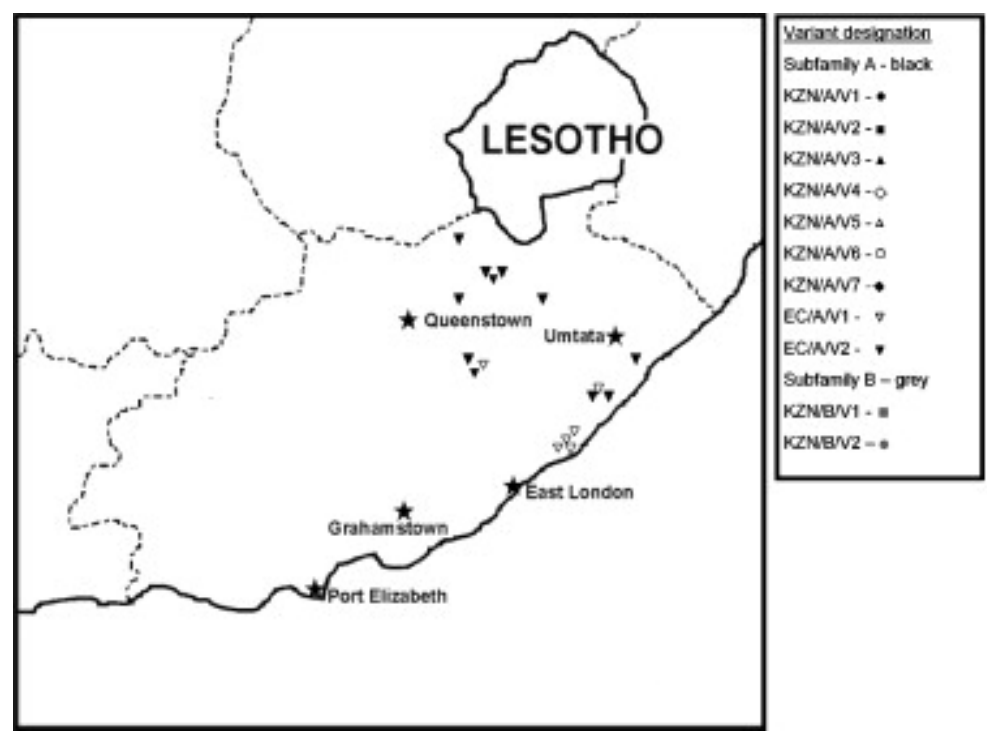

Fig. 5. A map of the Eastern Cape (EC) province demonstrating the approximate geographic origin of rabies viruses that were sequenced during the course of this study. Symbols correspond to those that were used to indicate the respective viral groupings on the phylogenetic tree in Fig. 3. 


\section{Table 2.}

Reference rabies virus sequences included in this analysis (Sabeta et al., 2003 and Nel et al., 2005)

\begin{tabular}{|c|c|c|c|c|c|c|c|}
\hline Number & $\begin{array}{l}\text { Lab reference } \\
\text { number }^{\mathrm{a}}\end{array}$ & Species $^{b}$ & $\begin{array}{l}\text { Country of } \\
\text { isolation }\end{array}$ & $\begin{array}{l}\text { Year of } \\
\text { isolation }\end{array}$ & Locality & $\begin{array}{l}\text { Coordinates } \\
\text { (longitude-latitude) }\end{array}$ & $\begin{array}{l}\text { Genbank accession } \\
\text { number }\end{array}$ \\
\hline 1 & dgA95.755 & Canine & South Africa & 1995 & Amanzintoti & $30^{\circ} 54^{\prime}-30^{\circ} 04^{\prime}$ & AF303081 \\
\hline 2 & dg373.97 & Canine & South Africa & 1997 & Baberton & $31^{\circ} 48^{\prime}-25^{\circ} 42^{\prime}$ & AF303069 \\
\hline 3 & $\operatorname{dg} 224.98$ & Canine & South Africa & 1998 & Ermelo & $29^{\circ} 59^{\prime}-26^{\circ} 31^{\prime}$ & AF177098 \\
\hline 5 & j596.99 & C. mesomelas & South Africa & 1999 & Piet Retief & $31^{\circ} 12^{\prime}-29^{\circ} 33^{\prime}$ & AF303063 \\
\hline 5 & $\mathrm{~d} 21057$ & Canine & Zimbabwe & 1992 & Muzarabani & $31^{\circ} 12^{\prime}-16^{\circ} 19^{\prime}$ & AF177064 \\
\hline 6 & $\mathrm{~d} 21869$ & Canine & Zimbabwe & 1993 & Nyakasoro, Pfungwe & $32^{\circ} 15^{\prime}-16^{\circ} 49^{\prime}$ & AF177069 \\
\hline 7 & $\mathrm{~d} 22547$ & Canine & Zimbabwe & 1994 & Kumutsenzere, Masoso & $31^{\circ} 47^{\prime}-16^{\circ} 22^{\prime}$ & AF177070 \\
\hline 8 & d19385 & Canine & Zimbabwe & 1991 & Zaka & $31^{\circ} 34^{\prime}-20^{\circ} 12^{\prime}$ & AF303080 \\
\hline 9 & $\mathrm{~d} 24505$ & Canine & Zimbabwe & 1996 & Gutu & $31^{\circ} 10^{\prime}-19^{\circ} 37^{\prime}$ & AF177075 \\
\hline 10 & d16387 & Canine & Zimbabwe & 1986 & Zhombe & $29^{\circ} 22^{\prime}-18^{\circ} 41^{\prime}$ & AF177057 \\
\hline 11 & $\mathrm{~d} 21428$ & Canine & Zimbabwe & 1993 & Chipinge & $32^{\circ} 43^{\prime}-20^{\circ} 22^{\prime}$ & AF177065 \\
\hline 12 & $\mathrm{j} 23275$ & C. adustus & Zimbabwe & 1995 & Bindura & $31^{\circ} 21^{\prime}-16^{\circ} 56^{\prime}$ & AF177089 \\
\hline 13 & $\mathrm{~d} 24465$ & Canine & Zimbabwe & 1996 & Wendza & $31^{\circ} 43^{\prime}-18^{\circ} 52^{\prime}$ & AF177074 \\
\hline
\end{tabular}




\begin{tabular}{|c|c|c|c|c|c|c|c|}
\hline Number & $\begin{array}{l}\text { Lab reference } \\
\text { number }^{\mathrm{a}}\end{array}$ & Species $^{\mathrm{b}}$ & $\begin{array}{l}\text { Country of } \\
\text { isolation }\end{array}$ & $\begin{array}{l}\text { Year of } \\
\text { isolation }\end{array}$ & Locality & $\begin{array}{l}\text { Coordinates } \\
\text { (longitude-latitude) }\end{array}$ & $\begin{array}{l}\text { Genbank accession } \\
\text { number }\end{array}$ \\
\hline 14 & j17711 & C. mesomelas & Zimbabwe & 1988 & Turk Mine & $30^{\circ} 49^{\prime}-19^{\circ} 27^{\prime}$ & AF177087 \\
\hline 15 & j306.98 & C. mesomelas & South Africa & 1998 & Warmbaths & $28^{\circ} 07^{\prime}-24^{\circ} 51^{\prime}$ & AF177105 \\
\hline 16 & j673.99 & C. mesomelas & South Africa & 1999 & Potgietersrus & $28^{\circ} 36^{\prime}-22^{\circ} 43^{\prime}$ & AF303061 \\
\hline 17 & j717.99 & C. mesomelas & South Africa & 1999 & Pietersburg & $29^{\circ} 29^{\prime}-23^{\circ} 42^{\prime}$ & AF303064 \\
\hline 18 & j23374 & C. mesomelas & Zimbabwe & 1995 & Bulawayo & $28^{\circ} 47^{\prime}-20^{\circ} 15^{\prime}$ & AF177091 \\
\hline 19 & j631 & C. mesomelas & South Africa & 1999 & Soutpansberg & $30^{\circ} 05^{\prime}-22^{\circ} 03^{\prime}$ & AF303060 \\
\hline 20 & m669.90 & $\begin{array}{l}\text { Cynictis } \\
\text { penicillata }\end{array}$ & South Africa & 1990 & Grootgewaagd & $29^{\circ} 52^{\prime}-26^{\circ} 42^{\prime}$ & AF079907 \\
\hline 21 & o377.99 & O. megalotis & South Africa & 1999 & Gordonia & $30^{\circ} 28^{\prime}-18^{\circ} 09^{\prime}$ & AF177119 \\
\hline 22 & 0414.96 & O. megalotis & South Africa & 1996 & Beaufort West & $22^{\circ} 47^{\prime}-32^{\circ} 22^{\prime}$ & AF177112 \\
\hline 23 & o469.99 & O. megalotis & South Africa & 1999 & Kimberley & $24^{\circ} 27^{\prime}-28^{\circ} 47^{\prime}$ & AF303073 \\
\hline 24 & o491.98 & O. megalotis & South Africa & 1998 & Petrusburg & $25^{\circ} 29^{\prime}-29^{\circ} 23^{\prime}$ & AF303059 \\
\hline 25 & o491.98 & O. megalotis & South Africa & 1998 & Petrusburg & $25^{\circ} 29^{\prime}-29^{\circ} 23^{\prime}$ & AF303059 \\
\hline 26 & 0578.95 & O. megalotis & South Africa & 1995 & Strydenburg/Hopetown & $23^{\circ} 46^{\prime}-29^{\circ} 55^{\prime}$ & AF177113 \\
\hline
\end{tabular}




\begin{tabular}{|l|l|l|l|l|l|l|l|}
\hline Number & $\begin{array}{l}\text { Lab reference } \\
\text { number }\end{array}$ & Species & & $\begin{array}{l}\text { Country of } \\
\text { isolation }\end{array}$ & $\begin{array}{l}\text { Year of } \\
\text { isolation }\end{array}$ & - Locality & $\begin{array}{l}\text { Coordinates } \\
\text { (longitude-latitude) }\end{array}$ \\
\hline 27 & 0774.95 & O. megalotis & South Africa & 1995 & Carnarvon & $21^{\circ} 56^{\prime}-31^{\circ} 13^{\prime}$ & AF177115 \\
\hline
\end{tabular}

${ }^{a}$ Laboratory reference numbers are preceded by the following symbols to indicate the host species of isolation, dog (dg, d), bat eared fox (o), jackal (j).

${ }^{\mathrm{b}}$ We use the term 'canine' to refer to domestic dogs (C. familiaris). 


\section{Discussion}

All the viruses in our panel were found to belong to the canid RABV biotype. The fact that the mongoose RABV biotype was not encountered was not unexpected, as the Drakensberg Mountains, a massive range on the western and northern border regions of the $\mathrm{KZN}$ and $\mathrm{EC}$ provinces, respectively, provides an effective barrier to the natural movement of wildlife from the mongoose rabies endemic regions of the central plateau. Rabies viruses in the KZN province segregated into two major groups (subfamilies) and the distribution pattern of the two subfamilies in their respective regions of isolation suggests that they represented independent epidemic fronts. The fact that the majority of viruses from the KZN province clustered into subfamily A, led us to believe that this lineage was representative of the present day core of the epidemic. Within this subfamily, which was not monophyletic, the order of the divergence events together with geographic distribution, suggested that rabies was likely to have been disseminated via the translocation of infected dogs. For instance, the order of the divergence events and geographic distribution for cluster KZN/A/V1-KZN-A/V3 and KZN/A/V4 as elucidated in the Section 3, was in accordance with an epidemic that had spread sequentially southwards along the northern coastal regions of the province, whereas the simultaneous divergence of clusters KZN/A/7 and KZN/A/V5-KZN/A/V6 (isolated from southern $\mathrm{KZN}$ ), suggested that the group 1 common ancestor was also introduced independently into southern KZN during the same time period. These translocation events are likely to have included the motorized transportation of infected dogs. It is noteworthy that a major transport artery, the N2 highway, runs all along the eastern seaboard of KZN. We identified a single independent cluster, comprised of three viruses, from the southern border regions of KZN. However, we could subsequently demonstrate by sequence analysis of viruses from the bordering EC province that isolates belonging to this cluster formed part of two larger rabies cycles (cluster EC/A/V1 and EC/A/V2). Both these clusters have a wide distribution throughout the EC and one of these, EC/A/V2, had evidently spread northwards into southern KZN. Importantly, the order of the divergence events suggested that the EC cycles became established at an earlier time period than the present-day cycles of subfamily A, that were identified from the coastal regions of the KZN province (KZN group of subfamily A viruses) (Fig. 3). 
Viruses in the second major group (subfamily B) were isolated from the northern regions of KZN and presented as two clusters. The percentage intrinsic sequence divergence for the two clusters was very low, with many of the sequences in both groups being identical. This may suggest that these RABV cycles represent very recent introductions. Perhaps not surprisingly, this lineage was found to be related to viruses (dog and jackal) from a northerly bordering province (Mpumalanga). The importance of this observation lies in the implicated role that jackals may have in the epidemiology of the disease within northern KZN itself, and further suggested that this viral lineage may have an underestimated, broad distribution throughout the neighbouring Mpumalanga province. The northern region of the KZN province coincides with the known distribution of the black backed jackal, and boasts intensive agricultural activities such as game ranching and cattle farming (URL: http://agriculture.kzntl.gov.za.dae.index.aspx/?ID=4, [Accessed 21 April 2005]). Such activities have previously been shown to provide ideal ecological conditions for the proliferation of the said vector species, allowing them to reach a sufficient population density at which they are able to sustain virus transmission (Estes, 1992, Bingham and Foggin, 1993 and Bingham, 2005). It is clear that rabies surveillance and epidemiological study in this region of KZN should continue to investigate and monitor the involvement of this wildlife species, towards elucidating the radiation and independence and/or co-dependence of dog and jackal rabies cycles.

In an extension of our investigation, rabies viruses from the EC province of RSA were analyzed for the first time, and provided us with a better understanding of the epidemiological relationship that exists between the KZN epidemic and newly recognized rabies cycles in the EC province. It was apparent that the majority of viruses from the KZN and EC provinces belonged to a unique viral lineage (subfamily A), which was in general distinguishable from $\mathrm{RABV}$ isolates that were obtained from elsewhere in southern Africa. However, beyond the collateral distinction of the subfamily A collection of viruses, they were found to segregate into three distinct groups that were unique to either the KZN (KZN group of subfamily A viruses) or the EC (EC/A/V1, EC/A/V2) province. Cumulatively our findings suggested that the most recent common ancestor of this subfamily could have been introduced on more than one occasion into either of the 
KZN and EC provinces. This observation is not entirely consistent with suggestions that the outbreaks in KZN and the EC provinces were commonly introduced through the sequential southwards spread of the disease along the eastern coastal belt of South Africa (Bishop et al., 2003 and Swanepoel, 2005).

We believe that there is one likely explanation for the phylogenetic and regional divisions within the subfamily A lineage of KZN and EC viruses. Consider that two epidemics had occurred among dogs in the affected provinces in recent times. The first epidemic spread through the KZN province from 1964 to 1968 and apparently did not enter the EC province (Bishop et al., 2003 and Swanepoel, 2005), whereas the second epidemic, which started in 1976, is known to have spread as far southwards as the EC province, from where it was reported since 1987 (Bishop et al., 2003 and Swanepoel, 2005). We suggest that during the 1964-1968 epidemic, rabies did in fact spread as far southwards as the northern regions of the EC province, which it entered to initiate outbreaks among local populations of domestic dogs. Although the first epidemic in KZN province was brought under control, the focus in the EC province probably persisted in the locations bordering on KZN, which was a former apartheid homeland, the Transkei. Here, rabies surveillance would have been nonexistent throughout the intervening time period between the first and second epidemic, i.e. from the late 1960 's to 80 's. The persistence of the initial outbreak in the EC province (Transkei), and the reintroduction of the disease into the coastal regions of KZN during the second epidemic, could then account for the phylogenetic patterns that emerged in our analyses. Finally, the delineation of the geographical distribution of the respective EC and KZN sequence clusters in this analysis, allows us to comment on the bidirectional movement of variants across the border between the two provinces. For example, viruses belonging to the KwaZulu Natal group of subfamily A viruses were isolated from the EC, whereas an isolate belonging to cluster EC/A/V2 (primarily composed of viruses from EC), was also isolated from KZN. It is probable that the movement of these variants have been facilitated by the transport of infected dogs along the major N2 highway, which spans the border regions between the two provinces, highlighting the requirement for stricter control on animal movement along this stretch of highway. 
This study is one of the most comprehensive and detailed for any specific epidemic of dog rabies in Africa to date. Most importantly, molecular epidemiology allowed us to track and describe the emergence and continued expansion of rabies in a defined region of southern Africa. We were also able to investigate historical dogma, to highlight the potential future involvement of wildlife in the epidemic, and to generate a sequence database for future surveillance efforts. It is our contention that the factors driving the proliferation of rabies in South Africa also apply throughout the rest of southern Africa and the larger continent as a whole. As the toll of rabies in Africa continues to escalate, the need for a refined approach and a serious focus on rabies, which so greatly benefited Europe and the Americas, should not be negated.

\section{References}

Bingham, 2005 J. Bingham, Canine Rabies ecology in Southern Africa, Emerg. Infect. Dis. 11 (2005) (9), pp. 1337-1342.

Bingham and Foggin, 1993 J. Bingham and C.M. Foggin, Jackal rabies in Zimbabwe, Onderstepoort J. Vet. Res. 60 (1993), pp. 365-366.

Bishop et al., 2003 Bishop, G.C., Durrheim, D.N., Kloeck, P.E., Godlonton, J.D., Bingham, J., Speare, J., 2003. Rabies: guide for the medical, veterinary and allied professions. Rabies Advisory Group, South African Department of Agriculture and Health.

DAEA, 2005 Department of Agriculture and Environmental Affairs, KwaZulu-Natal. Available from URL: http://agriculture.kzntl.gov.za.dae.index.aspx?ID=4 [Accessed 21 April 2005].

Estes, 1992 R.D. Estes, The Behavior Guide to African Mammals, University of California Press (Ltd., London, England (1992). 
Hall, 1999 T.A. Hall, BioEdit: a user friendly biological sequence alignment editor and analysis program for Windows 95/98.NT, Nucleic Acids Symp. Ser. 41 (1999), pp. 95-98.

Hills and Bull, 1993 D.M. Hills and J.J. Bull, An empirical test of bootstrapping as a method for assessing confidence in phylogenetic analysis, Syst. Biol. 42 (1993), pp. 182192.

King, 1993 A.A. King, Monoclonal antibody studies on rabies related viruses, Onderstepoort J. Vet. Sci. 60 (1993), pp. 283-287.

Kloeck, 1993 P. Kloeck, Rabies control in KwaZulu Natal, Proceedings of the South and Eastern African Rabies Group International symposium Pietermaritzburg, South Africa, 29-30 April 1993 (1993).

Kumar et al., 2004 S. Kumar, K. Tamura and M. Nei, MEGA3: integrated software for molecular evolutionary genetics analysis and sequence alignment, Brief. Bioinform. $\mathbf{5}$ (2004), pp. 150-163.

Nadin-Davis, 2000 S.A. Nadin-Davis, Rabies and rabies-related viruses. In: R.C.A. Thompson, Editor, Molecular Epidemiology of Infectious Diseases, Oxford University Press, New York (2000).

Nel et al., 1997 L. Nel, J. Jacobs, J. Jaftha and C. Meredith, Natural spillover of a distinctly canidae-associated biotype of rabies virus into the expanded wildlife host range in Southern Africa, Virus Genes 15 (1997) (1), pp. 79-82.

Nel et al., 2005 L.H. Nel, C.T. Sabeta, B. Von Teichman, J.B. Jaftha, C.E. Rupprecht and J. Bingham, Mongoose rabies in southern Africa: a re-evaluation based on molecular epidemiology, Virus Res. 109 (2005), pp. 165-173. 
Nel et al., 1993 L.H. Nel, G.R. Thomson and B.F. Von Teichman, Molecular epidemiology of rabies in South Africa, Onderstepoort J. Vet. Sci. 60 (1993), pp. 301306.

Paez et al., 2003 A. Paez, N. Nunez, C. Garcia and J. Boshell, Molecular epidemiology of rabies epizootics in Columbia: evidence for human and dog rabies associated with bats, $J$. Gen. Virol. 84 (2003), pp. 795-802.

Perret, 2005 K. Perret, Rabies update (provincial report for KZN), Proceedings of the South and Eastern African Rabies Group International symposium, eighth meeting Windhoek, Namibia, 24-25 January 2005 (2005).

Randles, 2003 J. Randles, Provincial rabies report: KwaZulu Natal, South Africa, Proceedings of the South and Eastern African Rabies Group International symposium, Seventh meeting Eluzwini, Swaziland, 12-15 May 2003 (2003).

Rupprecht et al., 2002 C.E. Rupprecht, C.A. Hanlon and T. Hemachudha, Rabies reexamined, Lancet Infect. Dis. 2 (2002), pp. 327-343.

Sabeta et al., 2003 C.T. Sabeta, J. Bingham and L.H. Nel, Molecular epidemiology of canid rabies in Zimbabwe and South Africa, Virus Res. 91 (2003), pp. 203-211.

Sacramento et al., 1991 D. Sacramento, H. Bourhy and N. Tordo, PCR technique as an alternative method for diagnosis and molecular epidemiology of rabies virus, Mol. Cell. Probes 5 (1991), pp. 229-240.

Saitou and Nei, 1987 N. Saitou and M. Nei, The neighbourhood-joining method: a new method for reconstructing phylogenetic trees, Mol. Biol. Evol. 4 (1987), pp. 406-425. 
Swanepoel, 2005 R. Swanepoel, Rabies. In: J.A.W. Coetzer and R.C. Tustin, Editors, Infectious Diseases of Livestock with Special Reference to Southern Africa, Oxford University Press, Southern Africa, Cape Town (2005), pp. 1123-1182.

Swofford and Olsen, 1990 D.L. Swofford and G.J. Olsen, Phylogeny reconstruction. In: D.M. Hillis and C. Moritz, Editors, Molecular Systematics, Sinauer Associates, Sunderland, MA (1990).

Thompson et al., 1994 J.D. Thompson, D.G. Higgins and T.J. Gibson, CLUSTALW: improving the sensitivity of progressive multiple sequence alignment through sequence weighting, position-specific gap penalties and weight matrix choice, Nucleic Acids Res. 22 (1994), pp. 4673-4680.

Tordo and Kouknetzoff, 1993 N. Tordo and A. Kouknetzoff, The rabies virus genome: an overview, Onderstepoort J. Vet. Sci. 60 (1993), pp. 263-269.

Tordo et al., 1988 N. Tordo, O. Poch, A. Ermine and G. Keith, Primary structure of the leader RNA and nucleoprotein genes of the rabies genome: segmented homology with the VSV, Nucleic Acid Res. 14 (1988), pp. 2671-2683.

Tordo et al., 1986 N. Tordo, O. Poch, A. Ermine, G. Keith and F. Rougeon, Walking along the rabies genome: is the large G-L intergenic region a remnant gene?, Proc. Natl. Acad. Sci. 83 (1986), pp. 3914-3918.

Von Teichman et al., 1995 B.F. Von Teichman, G.R. Thomson, C.D. Meredith and L.H. Nel, Molecular epidemiology or rabies in South Africa: evidence for two distinct virus groups, J. Gen. Virol. 76 (1995), pp. 73-82.

WHO, 2004 WHO, 2004. WHO expert consultation on rabies. Technical Report Series, 931, World Health Organization, Geneva. 
Wunner, 2002 W.H. Wunner, Rabies virus. In: A.C. Jackson and W.H. Wunner, Editors, Rabies, Elsevier Science, London (2002), pp. 23-77.

${ }^{\text {许 }}$ The Genbank accession numbers for the sequences reported in this paper are DQ841403-DQ841549.

枝结We present the first molecular epidemiological evidence for the emerging distribution patterns of rabies in the coastal region of southern Africa, and resolve an epidemiological scenario that may well apply to much of the African continent.

Corresponding author. Tel.: +27 12420 3622; fax: +27 124203266 . 